\title{
Atmospheres and surfaces of small bodies and dwarf planets in the Kuiper Belt
}

\author{
E.L. Schaller ${ }^{\mathrm{a}}$ \\ Lunar and Planetary Laboratory, University of Arizona, Tucson, AZ 85721, USA
}

\begin{abstract}
Kuiper Belt Objects (KBOs) are icy relics orbiting the sun beyond Neptune left over from the planetary accretion disk. These bodies act as unique tracers of the chemical, thermal, and dynamical history of our solar system. Over 1000 Kuiper Belt Objects (KBOs) and centaurs (objects with perihelia between the giant planets) have been discovered over the past two decades. While the vast majority of these objects are small $(<500 \mathrm{~km}$ in diameter), there are now many objects known that are massive enough to attain hydrostatic equilibrium (and are therefore considered dwarf planets) including Pluto, Eris, MakeMake, and Haumea. The discoveries of these large objects, along with the advent of large (> 6-meter) telescopes, have allowed for the first detailed studies of their surfaces and atmospheres. Visible and near-infrared spectroscopy of KBOs and centaurs has revealed a great diversity of surface compositions. Only the largest and coldest objects are capable of retaining volatile ices and atmospheres. Knowledge of the dynamics, physical properties, and collisional history of objects in the Kuiper belt is important for understanding solar system formation and evolution.
\end{abstract}

\section{Introduction}

The existence of a belt of debris beyond Neptune left over from planetary accretion was proposed by Kuiper in 1951 [1]. Though Pluto was discovered in 1930, it took over sixty years for other Kuiper belt objects (KBOs) to be detected [2] and for Pluto to be recognized as the first known member of a larger population now known to consist of over 1000 objects (Fig. 1). Studies of the dynamics of these objects over the past fifteen years (see Malhotra et al. [3] and references therein) have led to many insights about the early history of the outer solar system. For example, Neptune's outward migration has left indelible traces on the orbits of many Kuiper belt objects. Interactions with Neptune also scatter some Kuiper belt objects toward the inner solar system where they become centaurs (objects with perihelia between the giant planets) and can then be further scattered by the giant planets to become short period comets $[4,5]$.

Kuiper belt objects have been classified into several categories based on their dynamics including plutinos and twotinos (objects in the $3: 2$ resonance and 2:1 resonance with Neptune respectively), classicals (objects with low eccentricities and inclinations), and scattered disk and hot classicals (objects that have high eccentricities and inclinations) (see Gladman et al. [6] for a full discussion of KBO dynamical classes).

While the orbits of Kuiper Belt objects have revealed much about the early dynamical history of the solar system, detailed physical and chemical studies of individual objects are beginning to yield valuable information about the formation and evolution of these bodies themselves. Though the

\footnotetext{
a e-mail: els@lpl . arizona . edu
}

This is an Open Access article distributed under the terms of the Creative Commons Attribution-Noncommercial License 3.0, which permits unrestricted use, distribution, and reproduction in any noncommercial medium, provided the original work is properly cited. 

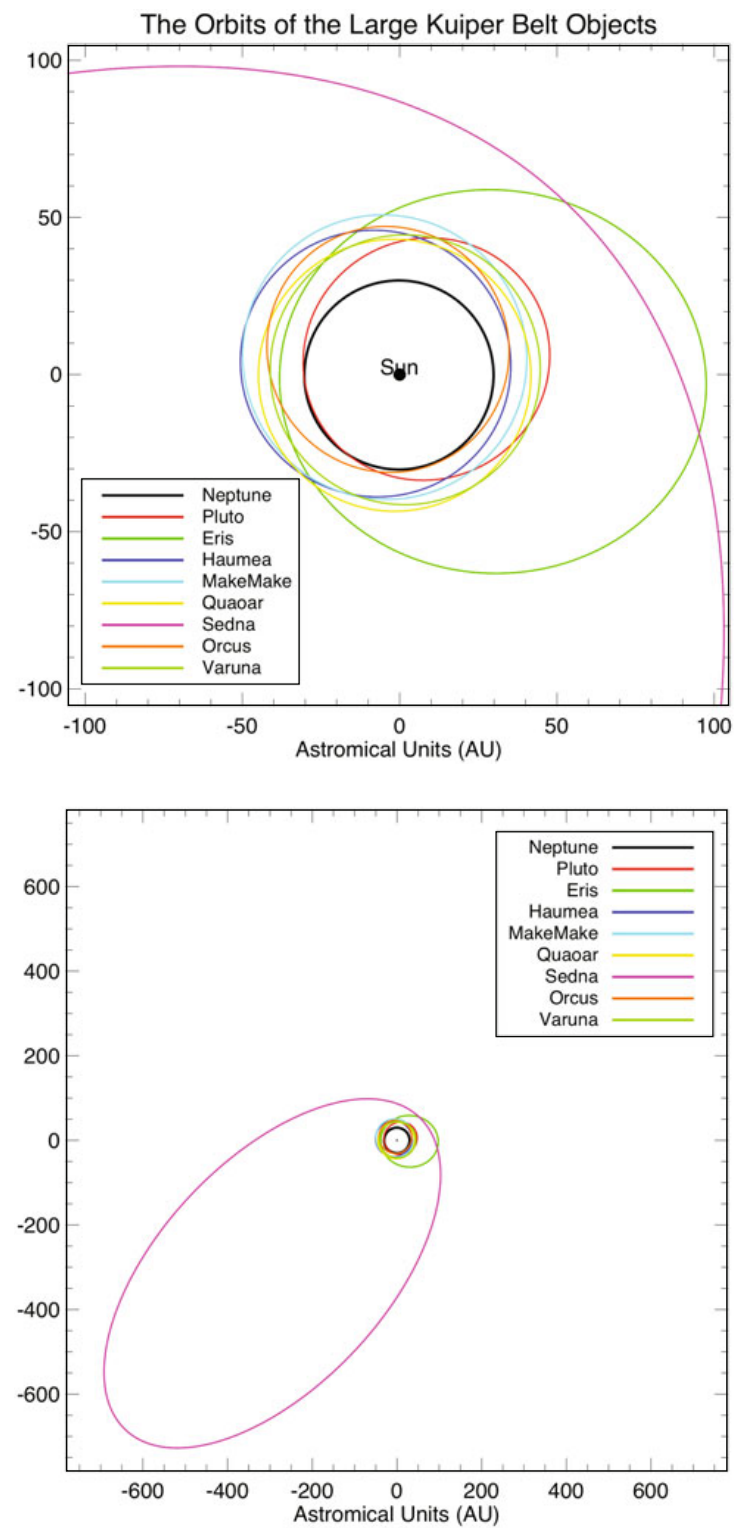

Fig. 1. The orbits of several of the largest Kuiper belt objects. Over 1000 objects are now known to orbit the sun in a belt between 30 and 50 Astronomical Units. The bottom panel shows the full orbit of Sedna, an extended scattered disk object. It is expected that many more objects in Sedna-like orbits will be eventually discovered.

surfaces of KBOs were initially expected to resemble dirty water-ice that had been slowly irradiated over 4.5 billion years, an initial diversity in their visible colours [7] gave the first indication that they had a wide range of surface compositions and histories.

The advent of large (> 6-meter aperture diameter) telescopes with sensitive visible and nearinfrared spectrographs, along with the new discoveries of Kuiper belt objects that rivalled or even exceeded the size of Pluto, led to an expansion of research on the surface compositions of these bodies [8-11].

In this chapter we discuss the different types of surfaces seen on objects in the outer solar system and how these surfaces relate to the atmospheres, dynamics, and collisional histories of individual objects. 


\section{Surfaces and atmospheres of KBOs}

Near infrared spectroscopy is particularly well suited for studying the surfaces of outer solar system bodies as many molecules expected to be present on these objects such as water ice, methane, nitrogen, ammonia, and higher order hydrocarbons have absorption bands in the 1-2.5 micron region.

Pluto and Neptune's satellite Triton (thought to be a captured Kuiper belt object due to its retrograde orbit) have surfaces covered in methane, molecular nitrogen and carbon monoxide ices [12-14]. These three ices $\left(\mathrm{N}_{2}, \mathrm{CO}\right.$, and $\left.\mathrm{CH}_{4}\right)$ all have significant vapour pressures at the low $(<50 \mathrm{~K})$ surface temperatures found on objects in the outer solar system and sublime to form Pluto and Triton's tenuous atmospheres. Other ices, volatile in the mid and inner solar system (such as ammonia, carbon dioxide, and water), are completely involatile at distances past $30 \mathrm{AU}$. Thus, in this discussion, volatile refers only to $\mathrm{N}_{2}, \mathrm{CO}$, and $\mathrm{CH}_{4}$ ices. Though $\mathrm{N}_{2}$ ice is the dominant surface constituent on both Pluto and Triton, $\mathrm{CH}_{4}$ ice is most easily detected due to several strong and broad absorption features in the near infrared. The near infrared spectra of Pluto and Triton resemble spectra of methane ice with small absorption features due to $\mathrm{N}_{2}$ and $\mathrm{CO}$ superposed.

Until recently these volatile-rich surfaces appeared to be unique in the outer solar system. Instead of volatile ices, the surfaces of all other smaller objects were covered with dark spectrally featureless material, with a few containing varying amounts of involatile water ice [8,15-17]. The recent discoveries of large KBOs that rival or exceed the size of Pluto with similar methane-dominated spectra have shown that these bodies are part of a new class of volatile-rich large objects in the outer solar system.

The explanation for why large objects contain volatile ices (and why other smaller objects in similar orbits do not) is clear when one considers the effects of atmospheric escape of volatile ices over the age of the solar system. Though all Kuiper belt objects likely formed with some fraction of volatile ices, only the largest bodies (diameter $>2000 \mathrm{~km}$ ) had enough gravity to prevent the thermal escape of their volatile ices to space over the age of the solar system. Smaller KBOs could not prevent the rapid sublimation and escape of all of their initial volatile ices [19]. Though these smaller objects likely contain volatile ices trapped in their interiors (as evidenced by the fact that Kuiper belt originating comets eject these volatiles) these ices cannot survive exposed on their surfaces in the outer solar system and thus are not detected.

\subsection{Large volatile-rich objects}

The spectrum of the largest KBO, Eris, like those of Pluto and Triton, is dominated by absorptions due to methane ice [20-22]. Though $\mathrm{N}_{2}$ has not been detected, shifts in the locations of the $\mathrm{CH}_{4}$ bands indicate that the $\mathrm{CH}_{4}$ is dissolved in $\mathrm{N}_{2}$ [23]. An additional factor conspiring to increase the difficulty of detecting $\mathrm{N}_{2}$ on Eris is that due to its great distance from the sun (97 AU), its molecular nitrogen is likely in the colder (alpha) form as opposed to the warmer (beta) form. In the alpha form, the weak 2.15 micron feature is significantly narrower and more difficult to detect. Though only methane has been detected, Eris likely contains all three volatile ices on its surface. Though Eris is near its aphelion and its volatile ices are completely frozen to its surface, it is possible that near its perihelion of $38 \mathrm{AU}$ (Table 1) its volatiles may sublime and form a thin atmosphere.

\subsection{Mid-sized volatile-rich objects}

In addition to the largest bodies, several mid sized ( $\sim 900-1500 \mathrm{~km}$ diameter) KBOs (Makemake, Quaoar and Sedna) also have spectra that show absorption features due to solid methane on their surfaces [25,26]. These bodies all have higher perihelia than Pluto and Triton and thus maintain colder temperatures throughout their orbits. These cold temperatures lower the vapour pressures of the volatile ices and have allowed their retention on the surfaces of these objects to the present day.

\subsubsection{MakeMake (2005 FY9)}

The spectrum of Makemake (Fig. 2) resembles the spectra of the largest KBOs and Triton in that it is dominated by absorptions due to methane ice $[25,34]$. However, in contrast to Pluto, Eris, and Triton, 
Table 1. Orbital Characteristics and surface compositions of outer solar system objects shown in Fig. 3.

\begin{tabular}{|c|c|c|c|c|}
\hline Name & $\begin{array}{c}\text { Diameter } \\
(\mathrm{km})\end{array}$ & $\begin{array}{c}\text { Perihelon } \\
(\mathrm{AU})\end{array}$ & $\begin{array}{c}\text { Aphelion } \\
(\mathrm{AU})\end{array}$ & $\begin{array}{c}\text { Known surface } \\
\text { volatiles }\end{array}$ \\
\hline Eris (2003 UB313) & $2400+/-100[27]$ & 37.8 & 97.6 & $\mathrm{CH}_{4}, \mathrm{~N}_{2}[20,21]$ \\
\hline Pluto & $2328+/-46[28]$ & 29.7 & 49.3 & $\mathrm{CH}_{4}, \mathrm{~N}_{2}, \mathrm{CO}[12,14]$ \\
\hline Charon & $1212+/-16[29]$ & 29.7 & 49.3 & $\mathrm{None}_{30]}[30]$ \\
\hline Triton & $2700[31]$ & 29.8 & 30.3 & $\mathrm{CH}_{4}, \mathrm{~N}_{2}, \mathrm{CO}[32]$ \\
\hline Haumea (2003 EL61) & $2000 \times 1500 \times 1000[10]$ & 35.1 & 51.5 & $\mathrm{None}^{2}[33]$ \\
\hline Sedna (2003 VB12) & $<1600[11]$ & 76.2 & 975 & $\mathrm{CH}_{4}, \mathrm{~N}_{2} ?[24]$ \\
\hline MakeMake (2005 FY9) & $1500+/-300[11]$ & 38.5 & 53.1 & $\mathrm{CH}_{4}[25,34]$ \\
\hline Quaoar (2002 LM60) & $1260+/-190[35]$ & 41.9 & 44.9 & $\mathrm{CH}_{4}[26]$ \\
\hline Orcus (2004 DW) & $950+/-70[11]$ & 38.5 & 48.3 & None $[36]$ \\
\hline
\end{tabular}

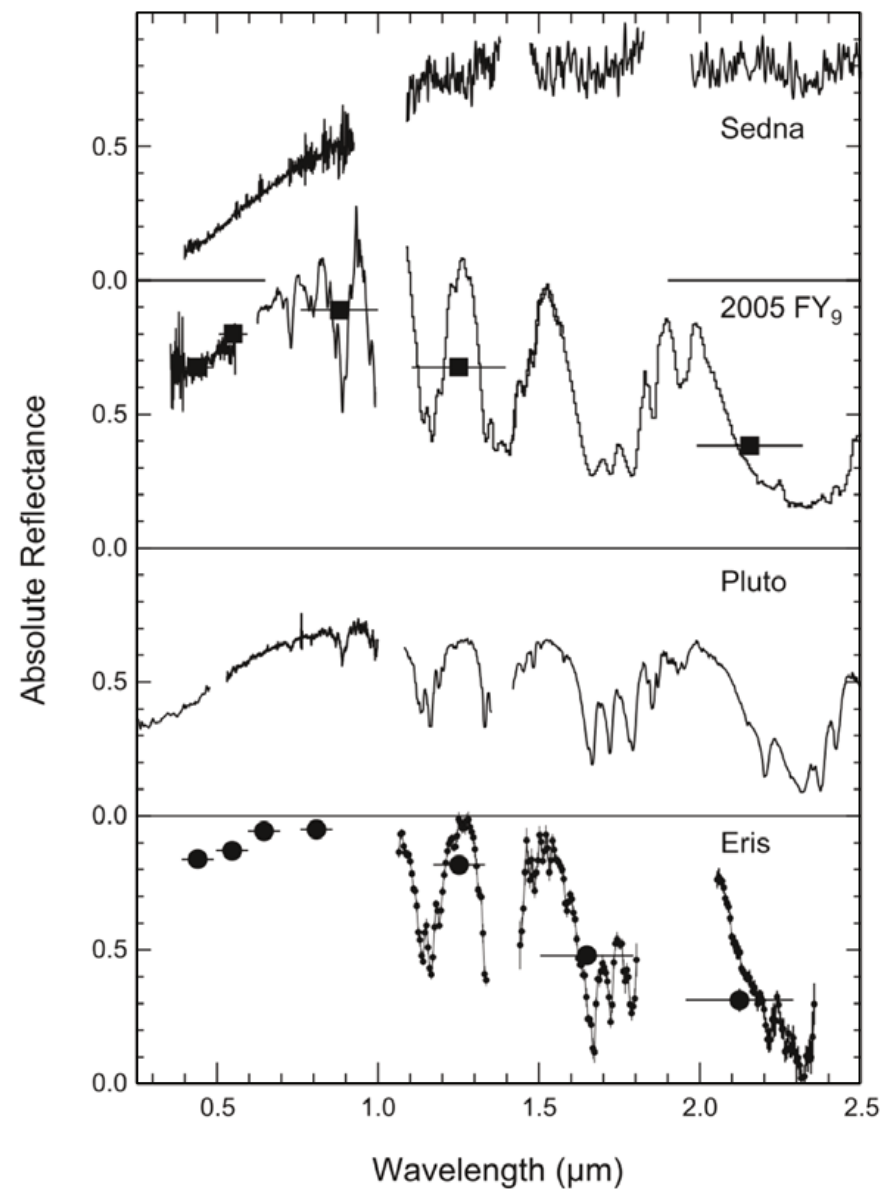

Fig. 2. Visible and Near Infrared spectra of the volatile-rich Kuiper belt objects showing broad absorption features due to methane ice (from the Solar System Beyond Neptune [18]).

the methane bands are much broader and more saturated on Makemake indicating that the methane is present in large (cm sized) grains. In addition, absorption features due to ethane ice and possibly other higher order hydrocarbons such as propane have also been detected [25]. The large grain sizes of methane and the presence of higher order hydrocarbons on the surface can all be explained if it is methane, rather than molecular nitrogen, that is the dominant surface constituent of Makemake [25]. This depletion of $\mathrm{N}_{2}$ can be understood in the context of the volatile loss model shown in Fig. 3 [19], MakeMake lies to the right of the methane line but to the left of the nitrogen line. On Pluto and 


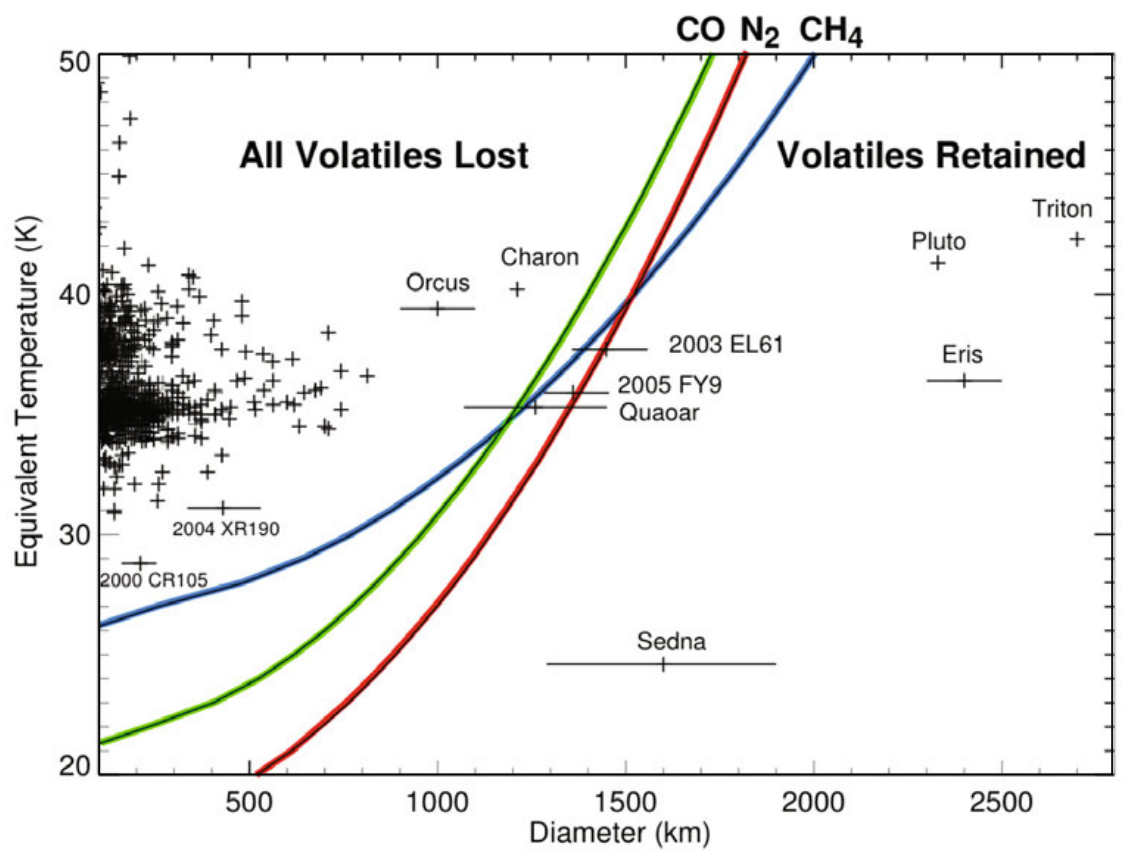

Fig. 3. Volatile loss model from Schaller \& Brown [19]. The lines show the diameter and temperature at which a body will have lost all of a given volatile ice over the age of the solar system. The vast majority of KBOs are too small and too hot to have retained any volatile ices on their surfaces. The largest and coldest bodies (Pluto, Triton, Eris and Sedna) should be capable of retaining volatile ices and potentially supporting atmospheres. 2003 EL61 (Haumea), 2005 FY9 (MakeMake) and 2002 LM60 (Quaoar) are in the transition region where they are potentially capable of retaining some volatile ices to the present day. Methane has been detected on MakeMake and Quaoar but not on Haumea. No volatile ices have been detected on any objects located to the left of the volatile loss lines in line with the model predictions.

Triton the production of higher order hydrocarbons is slowed due to the large amount of nitrogen on the surfaces of these bodies. When a methane molecule is photo-dissociated on Pluto or Triton, there are very few nearby methane molecules with which it can react to form higher order hydrocarbons. However, on Makemake, the large grains of methane provide abundant sites for such chemical reactions. Nitrogen is known to be at least half an order of magnitude less abundant on MakeMake than on Pluto [25].

\subsubsection{Quaoar (2002 LM60)}

The spectrum of Quaoar is dominated by absorptions due to crystalline water ice (see discussion of water ice objects in Section 2.3 but also contains several features in the infrared $(1.8,2.2,2.32,2.37$ microns) that have been attributed to the presence of a small amount of methane on its surface [26]. Quaoar's high mass [37] and distance from the sun indicate that it should be just barely capable of retaining volatile ices on its surface to the present day [19] (Fig. 3). The surface of Quaoar is consistent with that of a transition object between the large volatile rich objects and the small volatile free objects. Quaoar is presently losing the last of its volatiles via thermal escape. Models of Quaoar include water ice, a small amount of methane ice, and dark, featureless components. Tentative detections of additional features in the 2.2-2.4 micron range (not belonging to methane) may also indicate the presence of higher order hydrocarbons such as ethane [26].

\subsubsection{Sedna (2003 VB12)}

With a perihelion of $76 \mathrm{AU}$, the orbit of Sedna is far beyond the core of the Kuiper Belt (Fig. 1). Its distance means that it experiences extremely low temperatures throughout its orbit and is capable of 
retaining all of its volatile ices on its surface to the present day. However, due to its faintness, spectroscopy of Sedna is extremely difficult (Fig. 2). A tentative detection of methane has been reported as well as the suggestion of the presence of molecular nitrogen [24]. Other objects in similar orbits to Sedna (likely to be detected in the next generation sky surveys - see Section 3.2) should also be capable of retaining their volatile ices on their surfaces to the present day. Sedna is also one of the reddest objects in the solar system indicating that it likely contains many irradiation products on its surface.

\subsection{Non volatile-rich objects}

Most small and mid-sized $(<1500 \mathrm{~km}$ diameter) Kuiper belt objects and centaurs lay on a continuum between those whose spectra are essentially featureless to those whose spectra resembled pure crystalline water ice. Water ice is detected on these objects by the presence of the 1.5 and 2.0 micron absorption features (see Fig. 4). Its crystallinity is detected by the presence of an absorption feature at 1.65 microns.

To first order, the surfaces of small and midsized KBOs and centaurs can be described with a two-component model consisting of dark (likely carbon-rich) deposits and exposed crystalline water ice $[7,38]$. The spectra of these objects can be parameterized as a linear mixture between a featureless continuum component (with a variable slope) and crystalline water ice [7,38]. The fraction, $\mathrm{f}$, of water ice the spectra can then be compared with object size, orbital properties and object colour.

\subsubsection{Objects with featureless spectra $(\mathrm{f}<10 \%)$}

Figure 4 shows several objects whose spectra show no evidence for absorption features in the infrared. The vast majority of KBOs and centaurs smaller than $500 \mathrm{~km}$ diameter have surfaces with no identifiable spectral features even with moderately high signal-to-noise. These objects therefore have little to no fresh ices on their surfaces and are likely covered with refractory carbonaceous compounds formed from the irradiation of their surfaces over 4.5 billion years.

\subsubsection{Objects with moderate water ice spectra $(10 \%<\mathrm{f}<50 \%)$}

Several mid-sized (500-1500 km diameter) KBOs and several centaurs show moderate absorptions due to the presence of crystalline water ice on their surfaces (left frame of Fig. 4). Barkume et al. [38] found a weak correlation between object size and the fraction of water ice detected on the surfaces of the KBOs in their sample indicating that larger objects generally show stronger water ice absorption features. However, no such correlation was found for the Centaurs [38]. The degree of exposed water ice detected on the surfaces of all of these objects may be related to initial abundances or degree of differentiation, subsequent irradiation, impacts, or perhaps later extrusion of water ice onto the surface (cryo-volcanism).

Orcus is a plutino with a diameter approximately $1 / 3$ that of Pluto in a similar orbit [11] (Table 1 ). It is a particularly interesting object because in addition to moderate water ice absorption features, its spectrum shows a small absorption feature at 2.2 microns that has been interpreted to be due to the presence of either ammonia hydrate or methane ice $[36,40]$. Methane is not expected to survive to the present day on the surface of Orcus due to its small size $[19,41]$ so this feature has been generally interpreted as ammonia hydrate.

\subsubsection{Objects with strong water ice spectra ( $\mathrm{f}>80 \%)$ - The Haumea Collisional Family}

As discussed above, the near-infrared spectra of nearly all non-volatile rich Kuiper belt objects lie on a continuum between those whose spectra contain moderate amounts of water ice absorptions (water ice fraction less than 50\%) to those whose spectra are essentially featureless $[38,39,42]$. In 

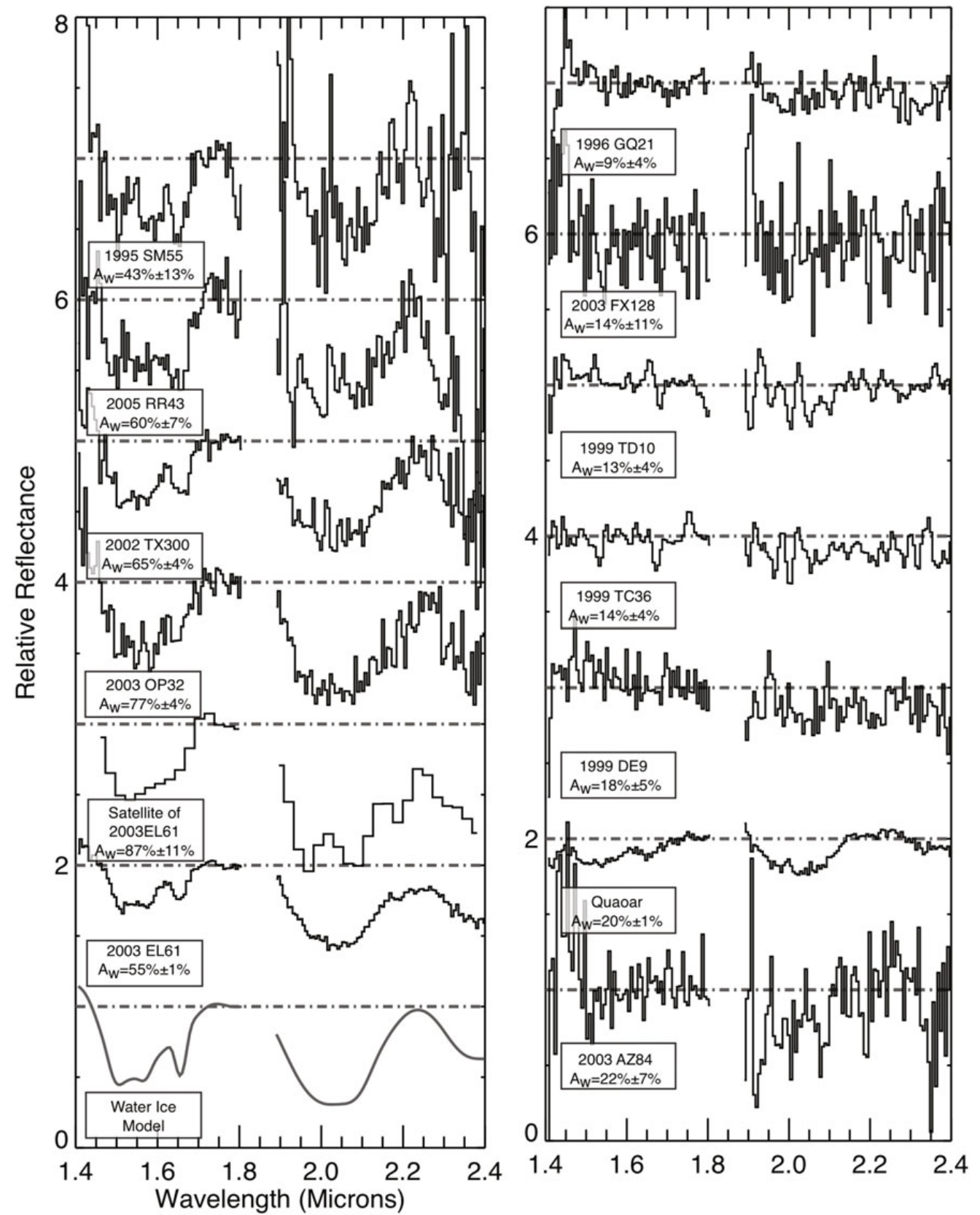

Fig. 4. Right panel: Near-Infrared spectra of several objects showing featureless (1996 GQ21, 2003 FX128, 1999 TD10) and moderate water ice spectra (1999 DE9, Quaoar, 2003 AZ84). Left Panel: Objects showing strong absorption features due to water ice at 1.5 and 2.0 microns (1995 SM55, 2005 RR43, 2002 TX300, 2003 OP32, and 2003 EL61 (Haumea) and its satellite). A pure water ice model spectrum is shown in the bottom left panel of the figure for comparison (Figure from [39]).

contrast, the near infrared spectra of Haumea (2003 EL61), its two satellites, and nine other small $\mathrm{KBOs}$ resemble laboratory spectra of pure crystalline water ice with modelled fractions of water ice greater than $80 \%[15,33,39,43-47]$. Remarkably, these objects are also relatively clustered in orbital element space. 


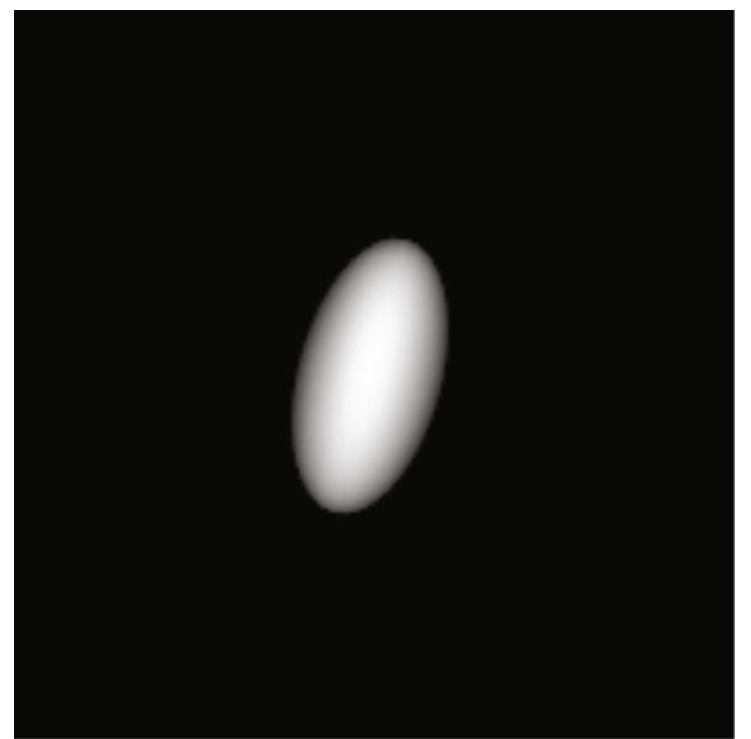

Fig. 5. The shape of Haumea (2003 EL61). Haumea's shape is due to its rapid (4-hour) rotation period that elongates it into a tri-axial ellipsoid. Haumea has a unique surface that looks remarkably similar to a spectrum of pure crystalline water ice (Figure courtesy M. Busch).

Haumea is perhaps one of the most intriguing objects in the solar system due to its fast rotation and odd shape. The shape of Haumea (a tri-axial ellipsoid) is due to its extremely fast rotation that acts to elongate it [10]. Haumea had been originally suggested to have experienced a massive collision that imparted its fast (4-hour) rotation, stripped off most of its icy mantle leaving it with a density close to rock $(2.7 \mathrm{~g} / \mathrm{cc})$, and formed its two satellites $[10,48,49]$. Due to the fact that the strong water ice spectral signature was only detected on Haumea, its satellites and objects orbiting close by, Brown et al. [39] concluded that the extremely water ice rich KBOs and the satellites of Haumea were in fact fragments of the icy mantle of the proto-Haumea that had been ejected during a massive collision. The strong water ice spectral signature has been used as a tracer of this collision. Haumea and its fragments are the first and only collisional family that has yet been detected in the Kuiper Belt.

\section{The future of outer solar system studies}

\subsection{Haumea mutual events}

In a stroke of astronomical good luck, the orbit of the inner satellite of Haumea is almost directly edgeon as viewed from the Earth and will be for approximately the next 5 years [50]. These mutual events between Haumea and its satellite Namaka will allow for detailed studies of this object that would be impossible to obtain any other way. Measuring the small decrease in brightness as the satellite passes directly in front of or behind Haumea will provide detailed information on the size, shape, and albedo of Haumea and its satellite as well as placing exquisite constraints on the masses and orbits of the Haumea system. Observations of mutual events of the Haumea system have already begun with telescopes all over the world.

\subsection{Large-scale survey telescopes}

Over the next decade, two dedicated survey telescopes (PanSTARRS and the Large Synoptic Survey Telescope (LSST)) will perform deep surveys of the sky and will find and track all moving objects. These surveys are expected to discover an order of magnitude more small bodies in the outer solar 
system than are currently known and may also find several new Pluto-sized objects. More objects will allow us to search for additional collisional families, characterize origins of Sedna-like objects, and test predictions volatile loss and retention.

\subsection{Next generation ground and space-based telescopes}

The next generation of large ground and space-based telescopes (the Thirty Meter Telescope (TMT), the Giant Magellan Telescope (GMT), and the James Webb Space Telescope (JWST)) will allow detailed spectroscopic studies of a much larger fraction of the known KBOs and their satellites. Currently, compositional information can only be obtained for the largest and brightest objects (visual magnitude $<21)$. The KBOs are the remnants of the icy building blocks that formed the giant planets and are analogous to the material in debris disks that are now being observed around other stars. Understanding the physical properties of these objects (both large and small) will be important for determining the origins and subsequent evolution of the objects in our solar system and in other planetary systems.

\subsection{New Horizons}

Finally, in July 2015, the New Horizons spacecraft will fly by the Pluto system. New Horizons carries a suite of instruments to characterize the Pluto-Charon system [51]. These instruments will map the surface compositions and temperatures of Pluto and Charon, characterize Pluto's atmosphere and escape rate, and search for rings and additional satellites. After its encounter with Pluto, New Horizons will also conduct similar investigations of one or more as-yet-to-be-determined Kuiper belt objects beyond Pluto.

\section{References}

1. G.P. Kuiper, Proc. Nat. Acad. Sci. 37, 1 (1951)

2. D. Jewitt, J. Luu, Proc. Nature 362, 730 (1993)

3. R. Malhotra, M.J. Duncan, H.F. Levison, Protostars and Planets IV 1231 (2000)

4. H.F. Levison, M.J. Duncan, Icarus 127, 13 (1997)

5. M.J. Duncan, H.F. Levison, Science 276, 1670 (1997)

6. B. Gladman, B.G. Marsden, C. Vanlaerhoven, Solar System Beyond Neptune (University of Arizona Press, 2008)

7. J. Luu, D. Jewitt AJ 112, 2310 (1996)

8. J. Luu, D. Jewitt ApJ 494, L117 (1998)

9. O.R. Hainaut, A.C. Delsanti, A\&A 389, 641 (2002)

10. D.L. Rabinowitz, et al. ApJ 639, 1238 (2006)

11. J. Stansberry, et al. Solar System Beyond Neptune (University of Arizona Press, 2008)

12. D.P. Cruikshank, C.B. Pilcher, D. Morrison, Science 194, 835 (1976)

13. D.P. Cruikshank et al. Science 261, 742 (1993)

14. T.C. Owen et al. Science 261, 745 (1993)

15. R.H. Brown, D.P. Cruikshank, Y. Pendleton, ApJ 519, L101 (1999)

16. W.M. Grundy, M.W. Buie, J.R. Spencer, AJ 130, 1299 (2005)

17. C.A. Trujillo, M.E. Brown, D.L. Rabinowitz, T.R. Geballe, ApJ 627, 1057 (2005)

18. M.E. Brown, Solar System Beyond Neptune (University of Arizona Press, 2008)

19. E.L. Schaller, M.E. Brown, ApJ 659, L61 (2007)

20. M.E. Brown, C.A. Trujillo, D.L. Rabinowitz, ApJ 635, L97 (2005)

21. J. Licandro, W.M. Grundy, N. Pinilla-Alonso, P. Leisy, A\&A 458 L5 (2006)

22. C. Dumas et al. A\&A 471, 331 (2007)

23. F. Merlin et al. A\&A 137, 315 (2009)

24. M.A. Barucci et al. A\&A 439, L1 (2005)

25. M.E. Brown et al. AJ 133, 284 (2007)

26. E.L. Schaller, M.E. Brown, ApJ 670, L49 (2007) 
27. M.E. Brown, E. L. Schaller, H.G. Roe, D.L. Rabinowitz, C.A. Trujillo, ApJ 643, L61 (2006)

28. E.F. Young, R.P. Binzel, Icarus 102, 134 (1993)

29. A.A.S. Gulbis et al. Nature 439, 48 (2006)

30. M.E. Brown, W.M. Calvin, Science 287, 107 (2000)

31. B.A. Smith et al. Science 246, 1422 (1989)

32. D.P. Cruikshank, P.M. Silvaggio, ApJ 233, 1016 (1979)

33. C.A. Trujillo, M.E. Brown, K.M. Barkume, E.L. Schaller, D.L Rabinowitz, ApJ 655, 1172 (2007)

34. J. Licandro et al. A\&A 445, L35 (2006)

35. M.E. Brown, C.A. Trujillo, AJ 127, 2413 (2004)

36. C. de Bergh, A. Delsanti, G.P. Tozzi, E. Dotto, A. Doressoundiram, M.A. Barucci, A\&A 437, 1115 (2005)

37. W. Fraser, M.E. Brown, ApJ 714, 1547 (2010)

38. K.M. Barkume, M.E. Brown, E.L. Schaller, AJ 135, 55 (2008

39. M.E. Brown, K.M. Barkume, D. Ragozzine, E.L. Schaller, Nature 446, 294 (2007)

40. M.A. Barucci et al., A\&A 479, L13 (2008)

41. A. Levi, M. Podolak, Icarus 202681 (2009)

42. A. Guilbert, et al., Icarus 201, 272 (2009)

43. K.M. Barkume, M.E. Brown, E.L. Schaller, ApJ 640, L87 (2006)

44. F. Merin, A. Guilbert, C. Dumas, M.A. Barucci, C. de Bergh, P. Vernazza, A\&A 466, 1185 (2007)

45. E.L. Schaller, M.E. Brown, ApJ 684, L107 (2008)

46. N. Pinilla-Alonso, J. Licandro, V. Lorenzi, A\&A 489, 445 (2008)

47. W. Fraser, M.E. Brown, ApJ 695, L1 (2009)

48. P. Lacerda, D. Jewitt, N. Peixinho, AJ 135, 1749 (2008)

49. M.E. Brown et al. ApJ 639, L43 (2006)

50. D. Ragozzine, M.E. Brown, AJ 137, 4766 (2009)

51. H.A. Weaver, S.A. Stern, Solar System Beyond Neptune (University of Arizona Press, 2008) 